\title{
Diagnostic accuracy and therapeutic impact of endoscopic ultrasonography in patients with intermediate suspicion of choledocholithiasis and absence of findings in magnetic resonance cholangiography
}

\author{
Enrique Vázquez-Sequeiros ${ }^{1}$, Fernando González-Panizo-Tamargo ${ }^{2}$, Daniel Boixeda-Miquel ${ }^{1}$ \\ and José María Milicua ${ }^{1}$ \\ ${ }^{1}$ Department of Gastroenterology. Hospital Universitario Ramón y Cajal. Madrid, Spain. ${ }^{2}$ Unit of Endoscopy. \\ Department of Digestive Diseases. Hospital Universitario Quirón. Madrid, Spain
}

\begin{abstract}
Background: endoscopic ultrasonography (EUS) and magnetic resonance cholangiography $(\mathrm{MRC})$ are the elective tests in the diagnosis of choledocholithiasis. MRC is best accepted by patients, but its sensitivity might decrease in the evaluation of microlithiasis.

Aim: to evaluate the diagnostic accuracy and therapeutic impact of EUS in a prospective cohort of patients with intermediate suspicion of choledocolithiasis and no findings in MRC (normal MRC).

Material and methods: during a period of 24 months, all the patients with clinical intermediate suspicion of choledocholithiasis and normal MRC were included. Sensitivity, specificity, diagnostic accuracy, positive predictive value (PPV) and negative predictive value (NPV) of MRC and EUS were compared, and so their impact in the management of these patients.

Results: seventy six patients were evaluated (lithiasis in 30\% of them). Sensitivity and diagnostic accuracy of EUS (100\%, 92\%) were significantly higher than MRC values $(0 \%, 70 \%)(p<0.05)$. EUS findings (suspicion of choledocholithiasis) favored a significant change in therapeutic attitude (therapeutic ERCP was performed) in 38\% of the patients (in which MRC had ruled out the presence of choledocholithiasis, and so, ERCP had not been performed) $(p<0.05)$.

Conclusions: EUS allows the diagnosis of lithiasis in approximately $1 / 3$ of patients with intermediate suspicion of choledocholithiasis and normal MRC. EUS findings involve a significant change in the management of these patients; this supports the use of EUS in clinical practice.
\end{abstract}

Key words: Endoscopic ultrasonography. Magnetic resonance Cholangiography. Diagnostic accuracy. Therapeutic impact. Choledocholithiasis.

Received: 15-02-11.

Accepted: 29-04-11.

Correspondence: Fernando González-Panizo-Tamargo. Unit of Endoscopy. Department of Digestive Diseases. Hospital Universitario Quirón Madrid. c/ Diego de Velázquez, 1. 28223 Pozuelo de Alarcón, Madrid. Spain. e-mail: fernandopanizo@yahoo.es
Vázquez-Sequeiros E, González-Panizo-Tamargo F, Boixeda-Miquel $D$, Milicua JM. Diagnostic accuracy and therapeutic impact of endoscopic ultrasonography in patients with intermediate suspicion of choledocholithiasis and absence of findings in magnetic resonance cholangiography. Rev Esp Enferm Dig 2011; 103: 464-471.

\section{INTRODUCTION}

Cholelithiasis is a major gastrointestinal disease, and choledocholithiasis is one of its main complications (1). The prevalence of choledocholithiasis in patients who have undergone a previous cholecystecthomy due to symptomatic cholelithiasis, and in patients with acute biliary pancreatitis, is estimated in 5-10\% and 18-33\%, respectively (2). Diagnosis of choledocholithiasis is based on clinical signs and symptoms, serum markers of cholestasis and imaging tests -abdominal ultrasonography and even computed tomography $(\mathrm{CT})-(3)$. Since the introduction of endoscopic retrograde cholangiography (ERCP), it has been considered the gold standard for the diagnosis and eventually the nonsurgical therapeutic approach for choledocolithiasis (4). However, because of the associated risks -pancreatitis (1,3$6,7 \%)$, infection $(0,6-5 \%)$, hemorrhage $(0,3-2 \%)$ and perforation $(0,1-1,1 \%)-(5-7)$, at the present time, ERCP is almost exclusively reserved for therapeutic purpose $(8,9)$, and it has been replaced by less invasive tests, such as magnetic resonance cholangiography (MRC) and endoscopic ultrasonography (EUS), for the diagnosis of choledocolithiasis.

In order to establish the likelihood of choledocholithiasis, different algorithms have been developed considering clinical, analytical and radiological findings; these algorithms allow us to classify patients in high, intermediate, indeterminated 
and low risk for having choledocholithiasis (2,10-12), what helps us to determine the diagnostic and therapeutic management of these patients (Fig. 1). According to the risk (probability) of choledocholithiasis, the recommended attitude is: a) low-indeterminate probability: it is not advised to continue the study of the biliary tree (clinical monitoring); b) intermediate probability: in these cases, it is advised to perform non invasive tests (MRC and/or EUS) before ERCP (13); and c) high probability: therapeutic ERCP is recommended (14).

Higher availability and less invasiveness of MRC compared with EUS have favored its use to date. However, the increasing availability of EUS in the hospitals of our health system and recent studies suggesting a higher diagnostic accuracy of EUS compared to MRC in the diagnostic of small-size lithiasis (< $5 \mathrm{~mm}$ ), might force us to reconsider the role of EUS in the algorithm of management of these patients (15). To date, no controlled data are available in our health system.

The present study aims to evaluate whether performance of EUS in these patients results in clinical usefulness, allowing rescue misdiagnosed patients by MRC.

\section{AIM}

To study prospectively the diagnostic accuracy and the therapeutic impact of EUS in patients with intermediate suspicion of choledocholithiasis and normal MRC.

\section{METHODS}

\section{Study design}

This prospective study, approved by the Ethical Committee, was carried out in a third level hospital. During a period of 24 months, all patients that fulfilled the following criteria were considered candidates to participate in the study:

- Inclusion criteria: a) intermediate clinical risk of choledocholitiasis (patient with previous diagnosis of cholelithiasis with or without clinical signs/symptoms, dilated biliary tree and elevated levels of at least alkaline phosphatase and aspartate alanine transferase (ALT), in the absence of fever (body temperature $\geq 38{ }^{\circ} \mathrm{C}$ ) (Fig. 1) and MRC negative for choledocholithiasis in the previous 2 weeks; b) patient older than 18 years; and c) absence of contraindications for ERCP.

- Exclusion criteria: a) patients who refuse to take part in the study; and b) patients who have undergone previous ERCP with or without esphincterothomy.

Those patients who fulfilled these criteria were offered to have a EUS after previous MRC had shown absence of choledocholithiasis.

All explorations were performed by an experienced biliary echoendoscopist ( $>5$ years and $>5,000$ echoendo-

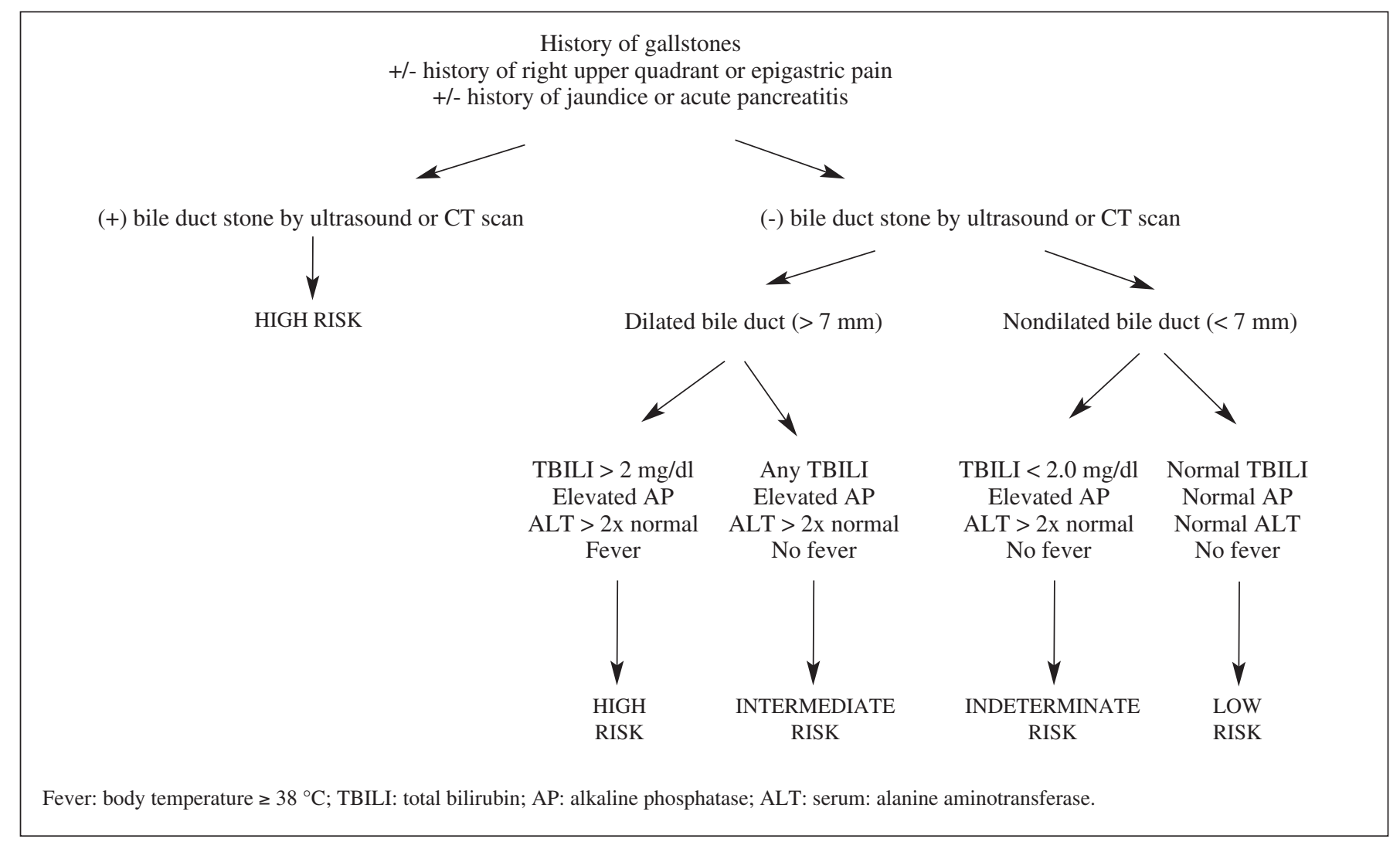

Fig. 1. Stratification of patients into risk groups based on clinical, biochemical and radiographic criteria -adapted from MI Canto et al. (12)-. 


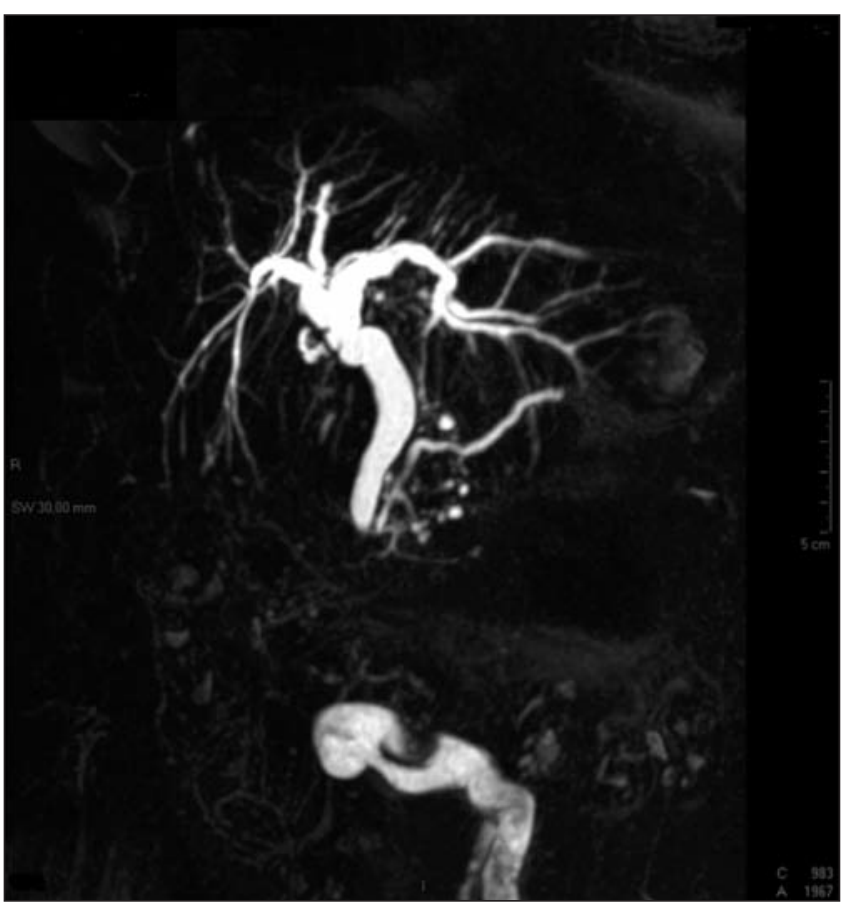

Fig. 2. Magnetic resonance cholangiography in a patient with intermediate risk of choledocholithiasis. Bile duct lithiasis are not identified.

scopies). Explorations were performed as an out-patient regime (excepting those patients admitted for other reasons), in a conventional endoscopy room and under conscious sedation performed by an endoscopist (midazolam, propofol, fentanyl and petidine with patient-adjusted dose). All the EUS exams were performed with an Olympus radial echoendoscope GF-UM160 (5-20 MHz, processor EUM60).

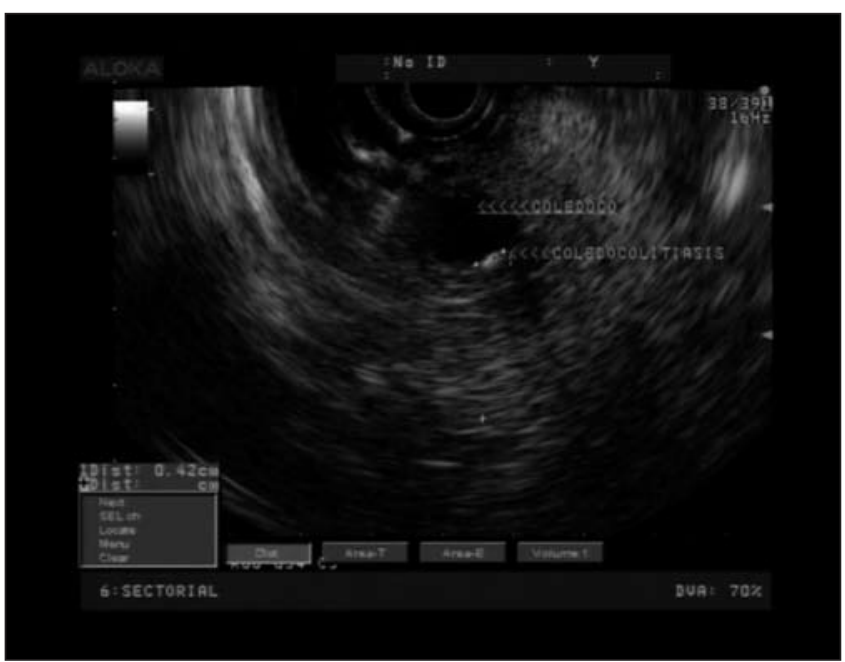

Fig. 3. Small-size lithiasis placed at the intrapancreatic bile duct. EUS performed after MRC in the same patient of the previous figure.

\section{Exam technique}

- MRC: magnetic resonance cholangiography was performed previously to EUS, out of the study protocol and according to the usual procedure and protocols for the MRC in the center of the study (1.5 Teslas equipment; gadopentetate dimeglumine (gadolinium) oral contrast using $\mathrm{T} 2$ high enhanced sequences and maximum projection intensity techniques, with following 3D reconstruction), as the usual diagnostic test in patients with suspicion of choledocholithiasis (Fig. 2).

- EUS: EUS was performed inside the study protocol. Echoendoscope was introduced to duodenal bulb, were the balloon was inflated to stabilize the echoendoscope; the air was then suctioned and water was instilled into the duodenal lumen. From this localization, proximal, medial and intrapancreatic choledochus was examined. Then, the echoendoscope was introduced into the second portion of the duodenum, were previously described maneuvers were performed again to evaluate the presence of lithiasis in this area (Fig. 3).

\section{Gold standard}

The presence of choledocholithiasis was determined by the following gold standard: a) when EUS agrees with MRC findings (absence of choledocholithiasis), both test were considered correct; and $b$ ) when there were a disparity between the findings of EUS and MRC (echoendoscopic suspicion of choledocholithiasis but normal MRC) the definitive diagnosis was based on therapeutic ERCP findings.

\section{Statistical analysis}

The statistical analysis of data obtained in the study was performed using the statistical software JMP 7.0.2 (SAS Institute Inc., North Carolina, USA). Descriptive analysis of the variables was performed: a) discrete variables: were described as percentage and $95 \%$ confidence interval; and b) continuous variables (normal distribution: mean, standard deviation and range) (non-normal distribution: median, interquartil range and range). Sensitivity, specificity, diagnostic accuracy, positive and negative predictive value for CRM and EUS were determined according to the usual methods (16). McNemar Test was used to compare MRC and EUS; this test is characterized by detecting, analyzing and comparing specifically discordant discrete matched data between both tests. Those diagnostic data that agree in both tests (whether correct or not), are ignored by $\mathrm{McNe}-$ mar Test (this is the reason why if MRC and EUS do not detect lithiasis, results of both procedures are correct according to the gold standard). Statistical significance level was established arbitrarily in $\alpha=0.05$. 


\section{RESULTS}

\section{Basal information of the patients included}

A total of 76 consecutive patients who met the study criteria were included during the period of time described above. Mean age was 67 years-old, and, as expected, there was a little predominance of women $(58 \%)$ in this cohort of patients. Basal features of this cohort of patients are shown in table I.

\section{Analysis of the diagnostic accuracy (EUS vs. MRC)}

EUS findings were compatible with choledocholithiasis in 29 of the 76 patients evaluated (38\%) (lithiasis size: median: $4 \mathrm{~mm}$, range: $3-8 \mathrm{~mm}$ ).

This diagnostic suspicion was confirmed by therapeutic ERCP in 23 of them (30\%), while in 6 patients no choledocholithiasis was found. In the remaining 53 patients, clinical monitoring was compatible with absence of choledocholithiasis, and that diagnosis was established (Fig. 4). Results from this study proved that EUS was significantly more sensitive and accurate than MRC for the diagnosis of choledocholithiasis in patients with intermediate likelihood for it (Table II).
Table I. Basal features of the patients

\begin{tabular}{lll}
\hline Variable & $n=76$ & $\%$ \\
\hline Age & & \\
Mean (years) \pm SD & $67 \pm 14.3$ & \\
$\quad$ Median & 69 & \\
Sex & & 58 \\
Male & $44 / 76$ & 42 \\
Female & $32 / 76$ & 28 \\
Tobacco & $21 / 76$ & 36 \\
Alcohol & $27 / 76$ & 18 \\
Cardiopathy & $14 / 76$ & 13 \\
Diabetes mellitus & $10 / 76$ & 7 \\
COPD & $5 / 76$ & \\
BMl & & \\
Mean $\left(\mathrm{kg} / \mathrm{m}^{2}\right) \pm S D$ & $33.8 \pm 4.3$ & \\
\hline
\end{tabular}

*SD: standard deviation; COPD: chronic pulmonary obstructive disease; BMI: body mass index.

\section{Therapeutic impact (USE vs. CRM)}

EUS findings (compatible with choledocholithiasis) favored a significant change in therapeutic attitude (therapeutic ERCP was performed) in $38 \%$ of the evaluated patients due to the suspicion of lithiasis in the biliary tree.

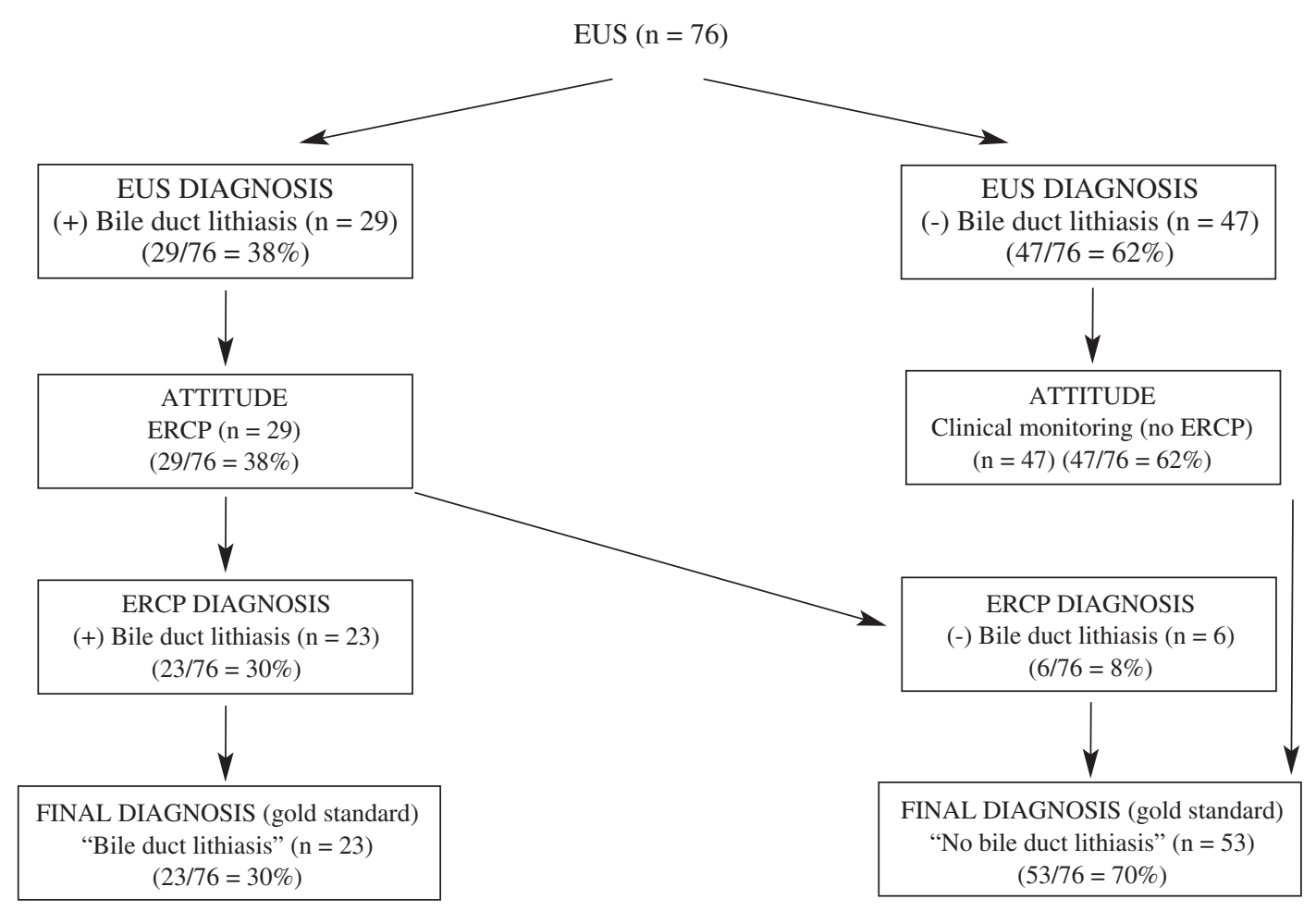

Fig. 4. Diagnostic algorithm in patients with intermediate risk of choledocholithiasis and normal MRC $(n=76)$. 
Table II. EUS and MRC values in the study of patients with intermediate risk of choledocholithiasis*

\begin{tabular}{llllll}
\hline & Sensitivity & Specificity & Accuracy & PPV & NPV \\
\hline EUS & & & & \\
$n \%$ & $23 / 23(100 \%)$ & $47 / 53(89 \%)$ & $70 / 76(92 \%)$ & $23 / 29(79 \%)$ \\
$95 \% \mathrm{Cl} *$ & $(88-100 \%)$ & $(77-96 \%)$ & $(84-97 \%)$ & $(60-92 \%)$ & $(94-100 \%)$ \\
\hline $\mathrm{MRC}$ & $0 / 23(0 \%)$ & $53 / 53(100 \%)$ & $53 / 76(70 \%)$ & $0 / 0(0 \%)$ & $53 / 76(70 \%)$ \\
$\mathrm{n} \%$ & $(0-12 \%)$ & $(94-100 \%)$ & $(58-80 \%)$ & $(0-100 \%)$ & $(58-80 \%)$ \\
$95 \% \mathrm{Cl}$ & 0.0001 & 0.04 & 0.003 & 0.0001 & 0.04
\end{tabular}

*Choledocholithiasis: $\mathrm{n}=23$ patients (lithiasis size: median: $4 \mathrm{~mm}$, range: $3-8 \mathrm{~mm}$ ); ${ }^{* * C l}$ : confidence interval.

It is important to highlight that, in all of these patients, MRC had previously excluded the presence of lithiasis, and consequently, had advised against performing an ERCP -EUS impact (38\%) vs. MRC impact (0\%) $(\mathrm{p}<0.05)-($ Fig. 5).

\section{DISCUSSION}

The significant amount of complications associated with ERCP has led to the almost definitive replacement of ERCP for less invasive techniques, such as MRC and EUS (2), in the diagnosis of the biliary pathology. Thus, ERCP is usually reserved for therapeutic interventions on the biliary tree $(8$,
9). Non invasive techniques (EUS and MRC) avoid ERCP risks $(12,13)$ and have a similar accuracy to that described for ERCP in detecting choledocholithiasis (85-95\%) (1720). In addition, some publications have suggested that performance of EUS previously to ERCP as a non-invasive screening test in patients with intermediate likelihood for choledocholithiasis, allows to avoid ERCP in $60-73 \%$ of this patients, and consequently, reduces the morbidmortality rate and costs (21-24).

Results from two metaanalysis (18-25) published in the last years comparing diagnostic accuracy of EUS and MRC conclude that no statistically significant differences between both techniques does exist for the detection of choledo-

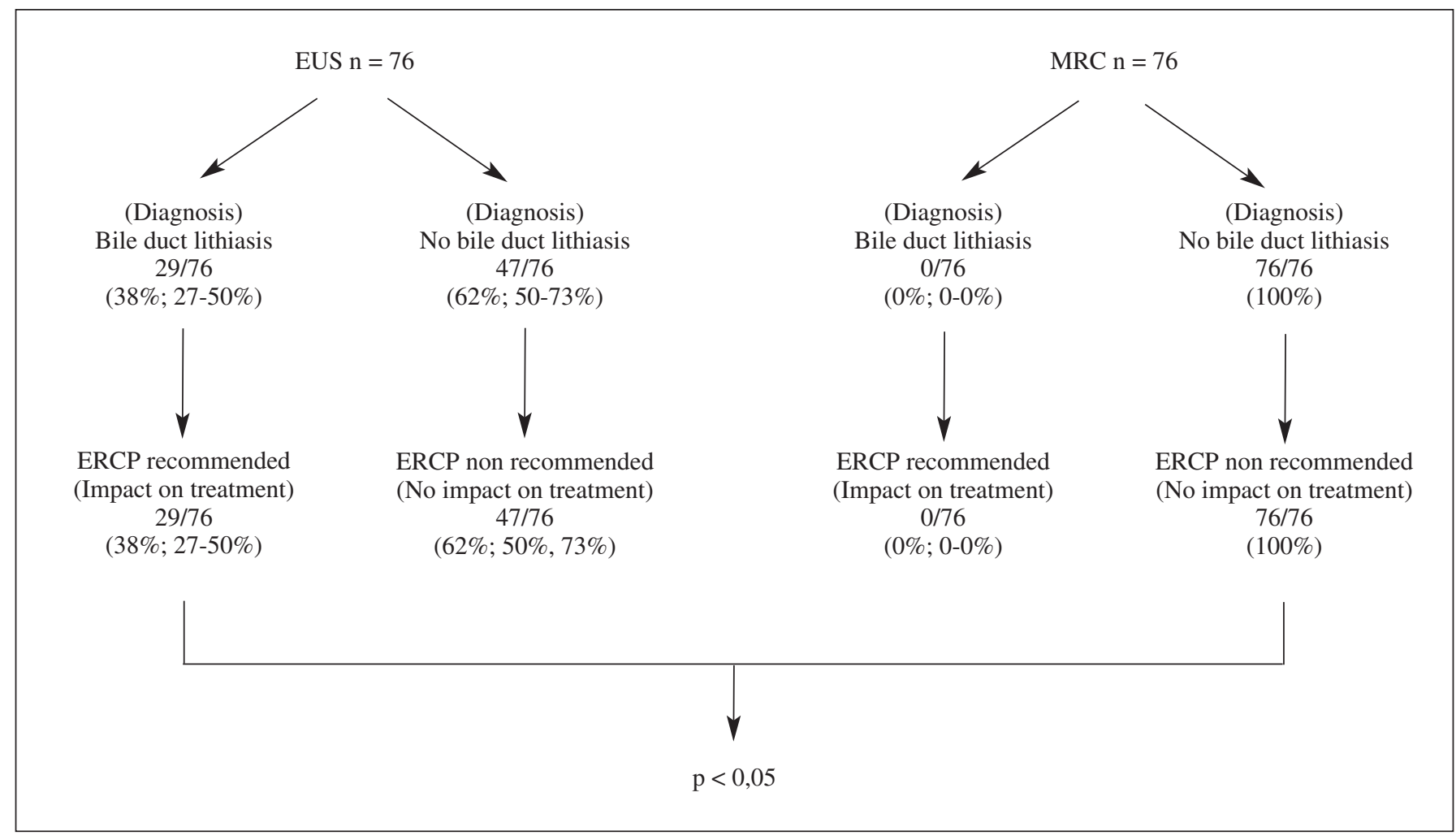

Fig. 5. Therapeutic impact of EUS in patients with normal MRC $(n=76)$. 
cholithiasis. However, increasing evidence suggests that EUS could be more sensitive for diagnosis of small-size lithiasis $(<5 \mathrm{~mm})(26-31)$. In this respect, it has been proven that, while in patients with small-size lithiasis, EUS diagnostic accuracy remains similar as in general studies (> 90\%) (26-28), sensitivity of MRC decreases significantly, and, in several studies, remains between 33 and $71 \%$ (2931 ). The reason of this decrease in MRC performance for the detection of small-size $(<5 \mathrm{~mm})$ lithiasis is not completely clarified. Endoscopists have suggested that the higher resolution of EUS compared with MRC could be responsible for the higher sensitivity of EUS in this subgroup of patients. However, those studies coming from radiologic field postulate that the higher sensitivity described for EUS is only real when non-high resolution magnetic resonance is performed (32). Results from our study suggest that even using high resolution equipments, there is an important number of patients with small-size lithiasis (median $4 \mathrm{~mm}$; range 3-8 $\mathrm{mm}$ in this study) detected by EUS that are not recognized by conventional MRC. In these patients, therapeutic ERCP is recommended in order to avoid future complications (cholangitis, pancreatitis,...) and this is only possible thanks to the EUS findings.

Though this is still a controversial issue, some scientific societies as The American Society for Gastrointestinal Endoscopy (ASGE) have suggested, in their clinical guides, to perform either EUS or MRC previously to ERCP in patients with intermediate likelihood for choledocholithiasis (2). Availability, experience of the medical team with both procedures in each workplace, and waiting lists may play an important role in the final decision between EUS and MRC. Likewise, patient opinion can force us to perform MRC instead of EUS because the first one does not involve the introduction of an endoscope under sedation. However, in patients with intermediate suspicion of choledocholithiasis and normal MRC, the results of the present study advise the performance of EUS to exclude smallsize lithiasis, since EUS allows the detection of a significant number of patients with lithiasis non-detected by MRC. These findings will determine a change in the therapeutic attitude in these patients. Specifically, in this study, from a total of 76 patients evaluated, EUS was able to detect choledocholithiasis in 29 (38\%) (in all of them, MRC performed in the previous two weeks had not detected pathology).

In these patients, therapeutic attitude was modified, as they were referred for ERCP with therapeutic intention due to echoendoscopic suspicion of choledocholithiasis. Echoendoscopic diagnosis was confirmed in the therapeutic ERCP in 23 of these patients. We highlight the fact that in all of them the size of tha lithiasis was $<5 \mathrm{~mm}$. Statistical analysis showed significant differences in favor of a higher therapeutic impact of EUS compared with MRC. Likewise, EUS proved to be significantly more sensitive and accurate than MRC. On the other hand, negative predictive value -NPV(the index that measures the certainty that when we say that there is no lithiasis, this affirmation is correct) of EUS is higher than NPV of MRC. Considering that, in our study, patients with negative EUS are no monitored anymore, we cannot exclude the possibility of false negative EUS results; however, this possibility would not affect either the results of the statistical comparisons or the statistically significant difference obtained. This is because McNemar statistical test compares discrete matched data as those provided by MRC and EUS, and only takes into account for the statistical analysis if there are discordant pairs in the carried out comparisons.

Thus, the high EUS NPV (100\% in this cohort of patients) is important because in the case that EUS does not find lithiasis, we can assure with a $100 \%$ of security (vs. $70 \%$ after MRC) that ERCP is not mandatory, particularly if we rule out dysfunction of Oddi sphincter. Data from our study agree with those previously published referred to this issue (26-31) (diagnostic accuracy of EUS in lithiasis $<5 \mathrm{~mm}$ significantly higher to MRC) and support the use of EUS in this type of patients. The original contribution from this study lies in the fact that it shows in our health system what foreign studies have previously suggested, the higher diagnostic capacity of EUS in these patients.

Finally, although it was not the aim of this study, it is reasonable to accept that the performance of EUS in these patients and the diagnosis small-size lithiasis, will allow the identification of patients with an increased risk of complications, which could be avoided by performing therapeutic ERCP (pancreatitis, cholangitis, hospital admissions, etc). This will potentially suppose cost savings (new admittance, repetition of tests, probable increasing in the emergency and seriousness of the patient condition, new therapeutic proceedings, intensive care,...).

However, we have to admit that this study have certain limitations. It represents the experience of a unique center, with its own characteristics, and consequently, the results cannot be generalized to other institutions (experience and technology available for EUS, etc.). On the other hand, we must recognize that, though high definition MRC was performed in all patients included in this study, it was carried out previously to the inclusion of the patients in the study. The fact that EUS was performed as a part of the study protocol (and not MRC) could hypothetically influence the result (meticulous performance of EUS because of the study). Nonetheless, what it is proved by the results of this study is that, in the clinical practice, MRC does not diagnose a far from negligible percentage of microlithiasis (30\% in this study) and EUS, on the other hand, seems to better identify these small-size lithiasis.

In conclusion, the results of this prospective and controlled study provide evidence favoring the use of EUS in patients with intermediate suspicion of choledocholithiasis and negative MRC. Higher sensitivity, accuracy and negative predictive value showed by EUS suggest that it should be indicated even in those patients with normal MRC. Confirmation of these results in multicentric studies would allow to generalize these conclusions. 


\section{REFERENCES}

1. Gallstones and laparoscopic cholecystectomy. NIH Consens Statement 1992; 10(3):1-28.

2. Maple JT, Ben-Menachem T, Anderson MA, Appalaneni V, Banerjee $\mathrm{S}$, Cash BD, et al. The role of endoscopy in the evaluation of suspected choledocholithiasis. ASGE Standards of Practice Committee, Gastrointest Endosc 2010;71(1):1-9.

3. Okuda K. Advances in hepatobiliary ultrasonography. Hepatology 1981;1(6):662-72.

4. NIH state-of-the-science statement on endoscopic retrograde cholangiopancreatography (ERCP) for diagnosis and therapy. NIH Consens State Sci Statements 2002;19(1):1-26.

5. Loperfido S, Angelini G, Benedetti G, Chilovi F, Costan F, De Berardinis F, et al. Major early complications from diagnostic and therapeutic ERCP: a prospective multicenter study. Gastrointest Endosc 1998; 48(1):1-10

6. Williams EJ, Taylor S, Fairclough P, Hamlyn A, Logan RF, Martin D, et al. Risk factors for complication following ERCP: results of a large-scale, prospective multi-center study. Endoscopy 2007;39(9):793801 .

7. Cotton PB, Garrow DA, Gallagher J, Romagnuolo J. Risk factors for complications after ERCP: a multivariate analysis of 11,497 procedures over 12 years. Gastrointest Endosc 2009;70(1):80-8.

8. Freeman ML, Nelson DB, Sherman S, Haber GB, Herman ME, Dorsher PJ, et al. Complications of endoscopic biliary sphincterotomy. N Engl J Med 1996;335(13):909-18.

9. Cotton PB, Lehman G, Vennes J, Geenen JE, Russell RC, Meyers WC, et al. Endoscopic sphincterotomy complications and their management: an attempt at consensus. Gastrointest Endosc 1991;37(3):383-93.

10. O'Toole D, Palazzo L. Choledocholithiasis -a practical approach from the endosonographer. Endoscopy 2006;38(Supl. 1):S23-9.

11. Trondsen E, Edwin B, Reiertsen O, Faerden AE, Fagertun H, Rosseland AR. Prediction of common bile duct stones prior to cholecystectomy: a prospective validation of a discriminant analysis function. Arch Surg 1998;133(2):162-6.

12. Canto MI, Chak A, Stellato T, Sivak MV Jr. Endoscopic ultrasonography versus cholangiography for the diagnosis of choledocholithiasis. Gastrointest Endosc 1998;47(6):439-48.

13. Williams EJ, Green J, Beckingham I, Parks R, Martin D, Lombard M; British Society of Gastroenterology. Guidelines on the management of common bile duct stones (CBDS). Gut 2008;57(7):1004-21.

14. Prat F, Meduri B, Ducot B, Chiche R, Salimbeni-Bartolini R, Pelletier G. Prediction of common bile duct stones by noninvasive tests. Ann Surg 1999;229(3):362-8

15. De Lédinghen V, Lecesne R, Raymond JM, Gense V, Amouretti M, Drouillard J, et al. Diagnosis of choledocholithiasis: EUS or magnetic resonance cholangiography? A prospective controlled study. Gastrointest Endosc 1999;49(1):26-31.

16. Motulsky H. In: Intuitive biostatistics. New York: Oxford University Press; 1995. p. 272-6.

17. Romagnuolo J, Bardou M, Rahme E, Joseph L, Reinhold C, Barkun AN. Magnetic resonance cholangiopancreatography: a meta-analysis of test performance in suspected biliary. Disease Ann Intern Med 2003;139(7):547-57.
18. Verma D, Kapadia A, Eisen GM, Adler DG. EUS vs MRCP for detection of choledocholithiasis. Gastrointest Endosc 2006; 64(2):248-54.

19. Tse F, Liu L, Barkun AN, Armstrong D, Moayyedi P. EUS: a metaanalysis of test performance in suspected choledocholithiasis. Gastrointest Endosc 2008;67 (2): 235-44.

20. Garrow D, Miller S, Sinha D, Conway J, Hoffman BJ, Hawes RH, et al. Endoscopic ultrasound: a metaanalysis of test performance in suspected biliary obstruction. Clin Gastroenterol Hepatol 2007;5(5):616-23.

21. Lee YT, Chan FK, Leung WK, Chan HL, Wu JC, Yung MY, et al. Comparison of EUS and ERCP in the investigation with suspected biliary obstruction caused by choledocholithiasis: a randomized study. Gastrointest Endosc 2008;67(4):660-8.

22. Polkowski M, Regula J, Tilszer A, Butruk E. Endoscopic ultrasound versus endoscopic retrograde cholangiography for patients with intermediate probability of bile duct stones: a randomized trial comparing two management strategies. Endoscopy 2007;39(4):296-303.

23. Liu CL, Fan ST, Lo CM, Tso WK, Wong Y, Poon RT, et al. Comparison of early endoscopic ultrasonography and endoscopic retrograde cholangiopancreatography in the management of acute biliary pancreatitis: a prospective randomized study. Clin Gastroenterol Hepatol 2005;3(12):1238-44.

24. Karakan T, Cindoruk M, Alagozlu H, Ergun M, Dumlu S, Unal S. EUS versus endoscopic retrograde cholangiography for patients with intermediate probability of bile duct stones: a prospective randomized trial. Gastrointest Endosc 2009;69(2):244-52.

25. Ledro-Cano D. Suspected choledocholithiasis: endoscopic ultrasound or magnetic resonance cholangio-pancreatography? A systematic review. Eur J Gastroenterol Hepatol 2007;19(11):1007-11.

26. Kondo S, Isayama H, Akahane M, Toda N, Sasahira N, Nakai Y, et al. Detection of common bile duct stones: comparison between endoscopic ultrasonography, magnetic resonance cholangiography, and helical-computed-tomographic cholangiography. Eur J Radiol 2005;54 (2):271-5.

27. Aubé C, Delorme B, Yzet T, Burtin P, Lebigot J, Pessaux P, et al. MR cholangiopancreatography versus endoscopic sonography in suspected common bile duct lithiasis: a prospective, comparative study. AJR 2005;184(1):55-62.

28. Sugiyama M, Atomi Y. Endoscopic ultrasonography for diagnosing choledocholithiasis: a prospective comparative study with ultrasonography and computed tomography. Gastrointest Endosc 1997;45 (2):143-6.

29. Zidi SH, Prat F, Le Guen O, Rondeau Y, Rocher L, Fritsch J, et al. Use of magnetic resonance cholangiography in the diagnosis of choledocholithiasis: prospective comparison with a reference imaging method. Gut 1999;44(11):118-22

30. Sugiyama M, Atomi Y, Hachiya J. Magnetic resonance cholangiography using half-Fourier acquisition for diagnosing choledocholithiasis. Am J Gastroenterol 1998;93(10):1886-90.

31. Boraschi P, Neri E, Braccini G, Gigoni R, Caramella D, Perri G, et al. Choledocholithiasis: diagnostic accuracy of MR cholangiopancreatography. Three-year experience. Magn Reson Imaging 1999; 17(9):124553.

32. McMahon CJ. The relative roles of magnetic resonance cholangiopancreatography (MRCP) and endoscopic ultrasound in diagnosis of common bile duct calculi: a critically appraised topic. Abdom Imaging 2008;33(1):6-9. 